\title{
The Correspondence Between Stochastic Linear Difference and Differential Equations
}

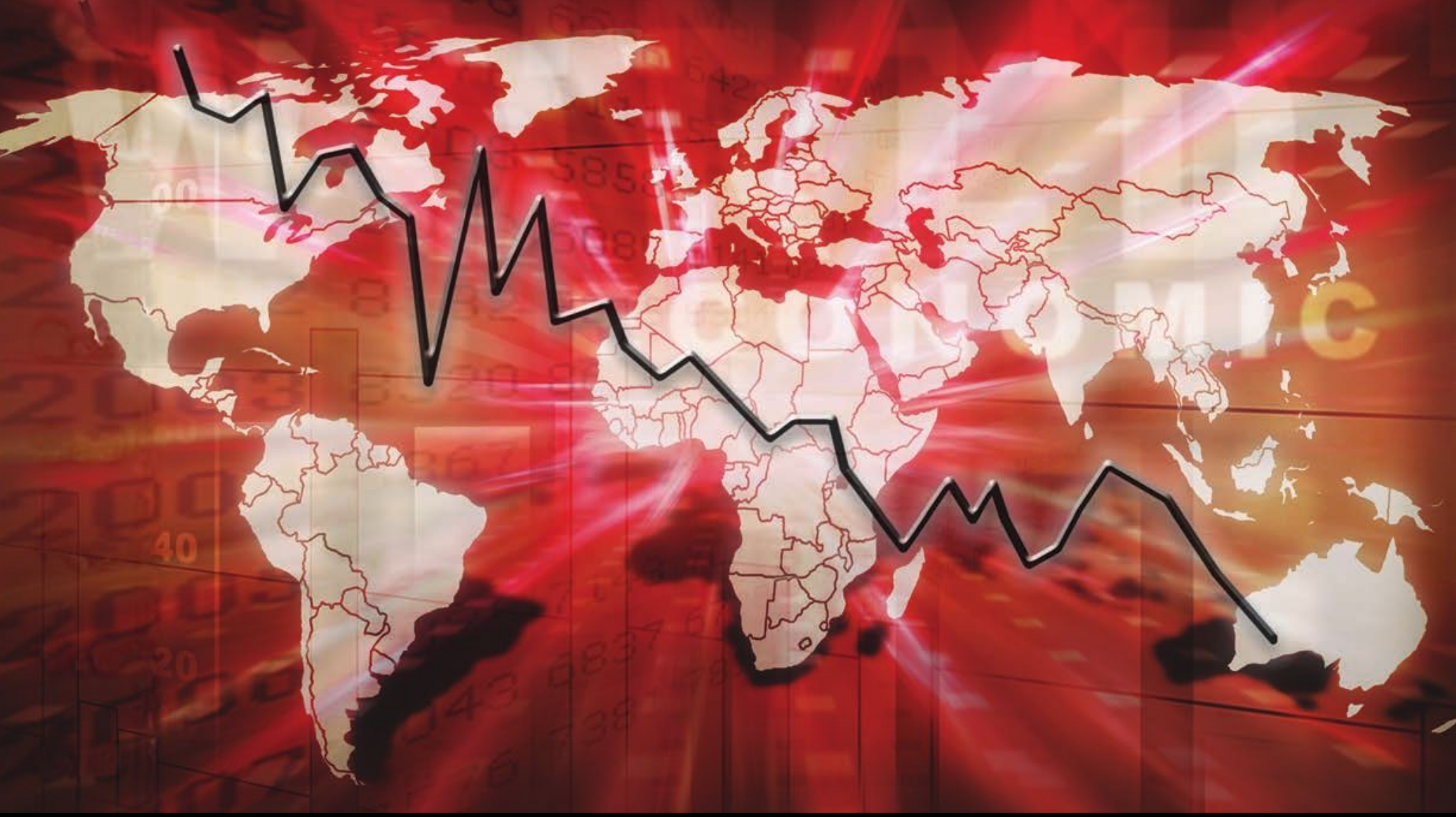

D.S.G Pollock, University of Leicester

Working Paper No. 1907 October 2019 


\title{
THE CORRESPONDENCE BETWEEN STOCHASTIC LINEAR DIFFERENCE AND DIFFERENTIAL EQUATIONS
}

\author{
By D.S.G. POLLOCK \\ University of Leicester \\ Email: stephen_pollock@sigmapi.u-net.com \\ Website: http://www.le.ac.uk/users/dsgp1/
}

The relationship between autoregressive moving-average (ARMA) models in discrete time and the corresponding models in continuous time is examined in this paper. The linear stochastic models that are commonly regarded as the counterparts of the ARMA models are driven by a forcing function that consists of the increments of a Wiener Process. This function is unbounded in frequency.

In cases where the periodogram of the data indicates that there is a clear upper bound to its frequency content, we propose an alternative frequencylimited white-noise forcing function. Then, there is a straightforward translation from the ARMA model to a differential equation, which is based of the principle of impulse invariance.

Whenever there is no perceptible limit to the frequency content, the translation must be based on a principle of autocovariance equivalence. On the website of the author, there is a computer program that effects both of these discrete-to-continuous translations.

\section{Introduction: The Discrete-Continuous Correspondence}

Modern communications technology relies on the correspondence between continuous signals and the discrete sequences that come from sampling the signals rapidly at regular intervals. Familiar examples of the technology are the analog-digital conversions of digital radio, digital sound recordings and digital television; but the domain of this technology is much wider.

The basis of digital technology is the sampling theorem of Nyquist $(1924,1928)$ and of Shannon (1949), which indicates that, if a signal is sampled with sufficient rapidity, then it can be reconstituted with complete accuracy from the sampled sequence.

The theorem is a commonplace amongst electrical engineers. It ought to be equally familiar to econometricians and statisticians and, in particular, to timeseries analysts, but it has been widely ignored.

This discrete-continuous equivalence began to be widely recognised at the end of the nineteenth century with the advent of the cinema. The cinema creates moving pictures from a sequence of fixed images projected in rapid succession. In the early days of the cinema, the succession of images was insufficiently rapid to convey an impression of smooth motion. The pictures tended to flicker; and, in popular parlance, we still refer to visiting the cinema as 'going to the flicks'.

There is a revealing picture by Marcel Duchamp, exhibited in the Paris Salon des Independents of 1912, which is titled A Nude Descending a Staircase. It exposes the paradox of the discrete-continuous correspondence; and it makes an allusion to the jerky motion of the early cinema. 
Occasionally, the true nature of motion pictures is revealed by an odd quirk that occurs when the rate of sampling is insufficient to convey a convincing impression of a rapid motion. Those of a certain age will have seen a depiction of a stagecoach fleeing its pursuers. They will have noticed the blurred impression of the wagon wheels. At times, these appear to be rotating slowly in the direction of travel. At other times, they seem to be stationary, and they may even, on occasion, appear to be moving backwards.

These are instances of the so-called problem of aliasing, whereby a motion that is too rapid to be captured by the sampling process is proxied by a much slower motion.

The Shannon-Nyquist sampling theorem is an adjunct of a Fourier analysis, which depicts a temporal trajectory as a weighted combination of trigonometric functions. The theorem indicates that, if the sampled sequence is fully to capture a continuous motion, then it is necessary that at least two observations should be made in the time that it takes for the trigonometric element of highest frequency to complete a single cycle. This rate of sampling, which corresponds to a signal frequency of $\pi$ radians per sampling interval, is the so-called Nyquist relative frequency.

If the frequencies within the signal exceed the Nyquist value of $\pi$, then there will be an irremediable loss of information and it will not be possible fully to reconstitute the signal from the sampled data. Conversely, if the maximum frequency within the signal is less than the Nyquist value, then the sampling is over-rapid and other problems can arise; but these problems ought, in principle, to be remediable.

\section{ARMA Estimation and the Effects of Over-rapid Sampling}

A problem can arise in the estimation of an ARMA model when the rate of sampling exceeds the maximum frequency within the signal. The problem can be illustrated with the deseasonalised quarterly data on U.S. gross domestic product (GDP) from which a trend has been extracted with the filter of Leser (1961) and of Hodrick and Prescott $(1980,1997)$ - see Figure 1. The problem is revealed by examining the periodogram of the data, which is a product of its Fourier transform.

The Fourier analysis expresses the detrended data sequence $y(t)=\left\{y_{t} ; t=\right.$ $0,1, \ldots, T-1\}$ as

$$
y(t)=\sum_{j=0}^{[T / 2]}\left\{\alpha_{j} \cos \left(\omega_{j} t\right)+\beta_{j} \sin \left(\omega_{j} t\right)\right\}=\sum_{j=0}^{T-1} \xi_{j} e^{\mathrm{i} \omega_{j} t}
$$

where $\omega_{j}=2 \pi j / T ; j=0, \ldots,[T / 2]$ are the Fourier frequencies, which are placed at regular intervals running from zero up to the Nyquist frequency $\pi$, or just short of it by a half interval. Here, $[T / 2]$ denotes the integer quotient of the division of $T$ by 2 . 


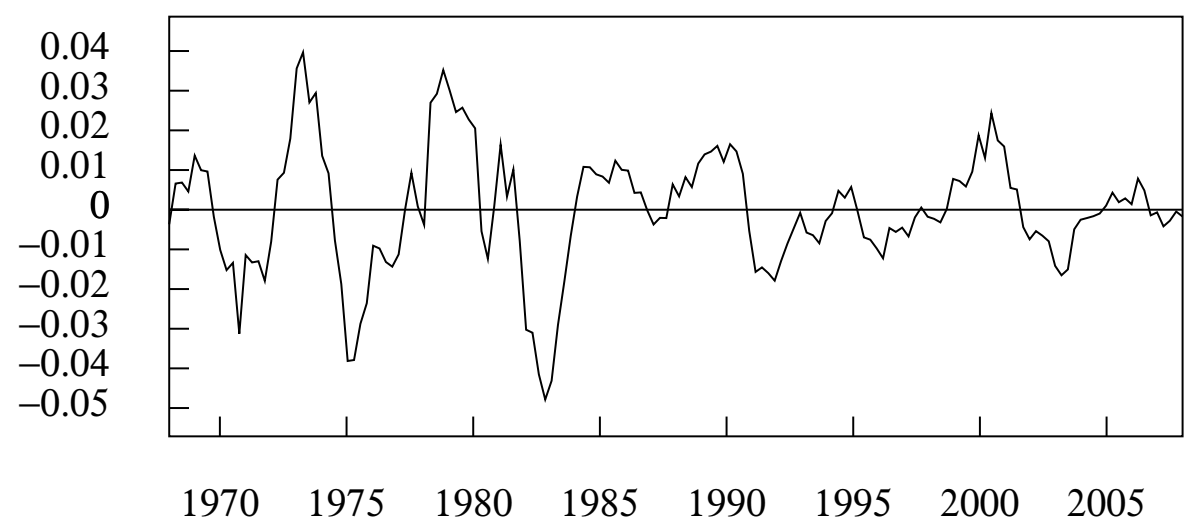

Figure 1. The deviations of the logarithmic quarterly index of real US GDP from an interpolated trend. The observations are from 1968 to 2007. The trend is determined by a Hodrick-Prescott (Leser) filter with a smoothing parameter of 1600.

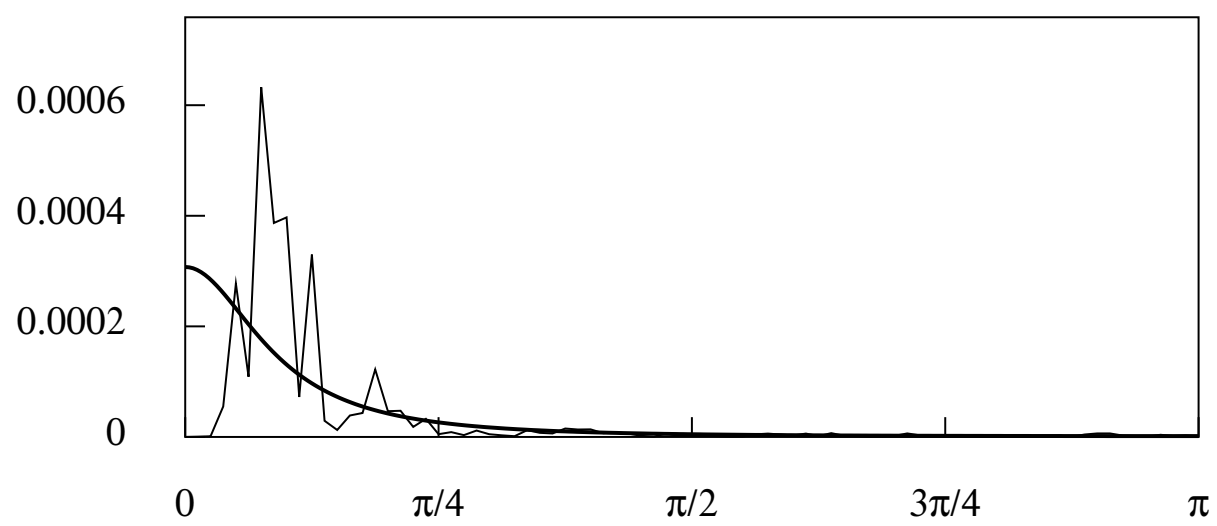

Figure 2. The periodogram of the data points of Figure 1 overlaid by the parametric spectral density function of an estimated regular $\mathrm{AR}(2)$ model.

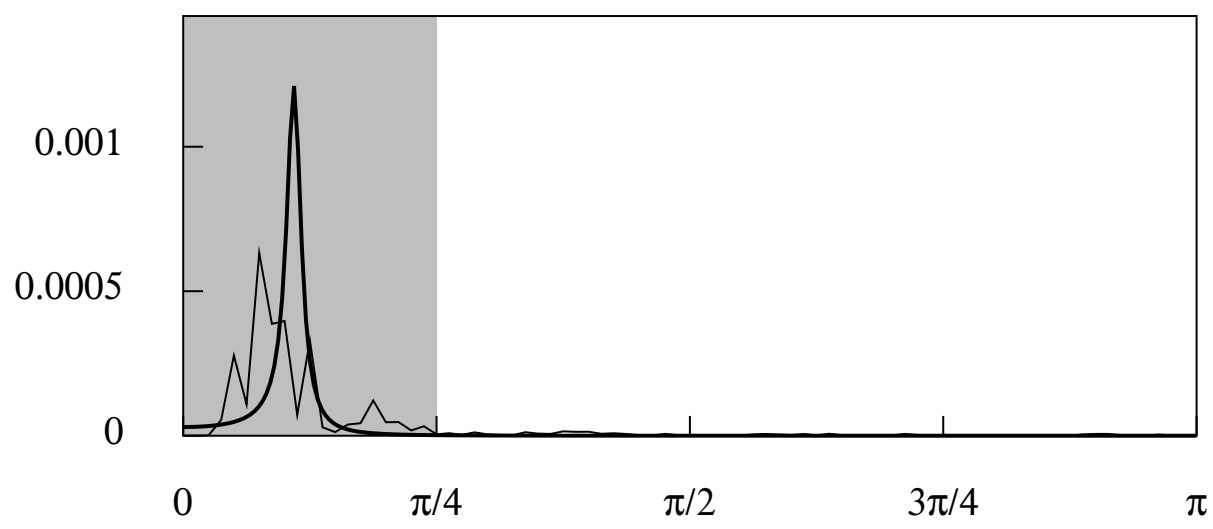

Figure 3. The periodogram of the data points of Figure 1 overlaid by the spectral density function of an $\operatorname{AR}(2)$ model estimated from frequency-limited data. 
The second expression, which employs complex exponential functions, arises from Euler's equations, whereby

$$
\cos \left(\omega_{j} t\right)=\frac{e^{\mathrm{i} \omega_{j} t}+e^{-\mathrm{i} \omega_{j} t}}{2} \text { and } \sin \left(\omega_{j} t\right)=\frac{-\mathrm{i}}{2}\left(e^{\mathrm{i} \omega_{j} t}-e^{-\mathrm{i} \omega_{j} t}\right) .
$$

Conversely, there are

$$
e^{\mathrm{i} \omega_{j} t}=\cos \left(\omega_{j} t\right)+\mathrm{i} \sin \left(\omega_{j} t\right) \quad \text { and } \quad e^{-\mathrm{i} \omega_{j} t}=\cos \left(\omega_{j} t\right)-\mathrm{i} \sin \left(\omega_{j} t\right),
$$

and it follow that $\exp \left\{-\mathrm{i} \omega_{j} t\right\}=\exp \left\{\mathrm{i} \omega_{T-j} t\right\}$. Also, $\xi_{j}=\left(\alpha_{j}-\mathrm{i} \beta_{j}\right) / 2$ and $\xi_{T-j}=$ $\left(\alpha_{j}+\mathrm{i} \beta_{j}\right) / 2$ for $j=0,1, \ldots,[T / 2]$. These results enable the two expressions of (1) to be reconciled.

The coefficients $\alpha_{j}, \beta_{j}$ are obtained by regressing the data on the ordinates of the trigonometric functions $\cos \left(\omega_{j} t\right), \sin \left(\omega_{j} t\right)$, where $t=0,1, \ldots, T-1$. It should be observed that, if the maximum frequency in the signal is less than $\pi$, then some of these coefficients will be zero valued.

The periodogram is the plot of the squared amplitudes $\rho_{j}^{2}=\alpha_{j}^{2}+\beta_{j}^{2}$, and it conveys a frequency-specific analysis of variance. That is to say

$$
V(y)=\frac{1}{T} \sum_{t}\left(y_{t}-\bar{y}\right)^{2}=\frac{1}{2} \sum_{j}\left\{\alpha_{j}^{2}+\beta_{j}^{2}\right\}=\frac{1}{2} \sum_{j} \rho_{j}^{2} .
$$

The periodogram of the detrended logarithmic quarterly index of real US GDP is depicted in Figures 2 and 3.

An attempt can be made to capture the business cycle dynamics by fitting an $\mathrm{AR}(2)$ model to the detrended data. The expectation is that the poles of the model, i.e. its autoregressive roots, will be a conjugate complex pair. The modulus of the roots should represent the damping characteristics of the business cycle and their argument should represent an angular velocity, which would indicate the average duration of the business cycle. The parametric spectrum of the fitted ARMA model should mimic the shape of the periodogram, with its peak in roughly the same position as that of the periodogram.

When the parametric spectrum of the estimated $\operatorname{AR}(2)$ model is superimposed on the periodogram in Figure 2, it becomes clear that, in place of the expected complex roots, there are two real-valued roots.

In diagnosing the problem, it is recognised that there are minor elements of noise affecting the data throughout the frequency interval running for the cut-off point of the spectral signature of the business cycle at $\omega_{c}=\pi / 4$ up to the Nyquist frequency of $\pi$. This noise is making a significant contribution to the variance of the data without greatly affecting the autocovariances at positive lags. As a result, the initial values, which determine the estimates of the autoregressive parameters, show an exaggerated rate of decline, or damping, which gives rise to the real-valued poles.

The appropriate recourse would seem to be to remove the noise from the data by suppressing the associated periodogram ordinates in the interval $(\pi / 4, \pi]$. When this is done, the estimation does deliver a pair of conjugate complex poles. However, in this case, the parametric spectrum in Figure 3 misrepresents the periodogram in another way. 
The poles are too close to the unit circle, i.e. their modulus is close to unity. The effect is to exaggerate the prominence of the spectral spike and to underestimate of the rate of damping. Also, it can be seen that the peak is displaced to the right, implying that the argument is an overestimate, which exaggerates of the frequency of the cycles. In consequence of the excessive rate of sampling, the initial autocovariances, which are too close to the origin, where the variance is to be found, are declining too slowly. Thus, the rate of damping is underestimated.

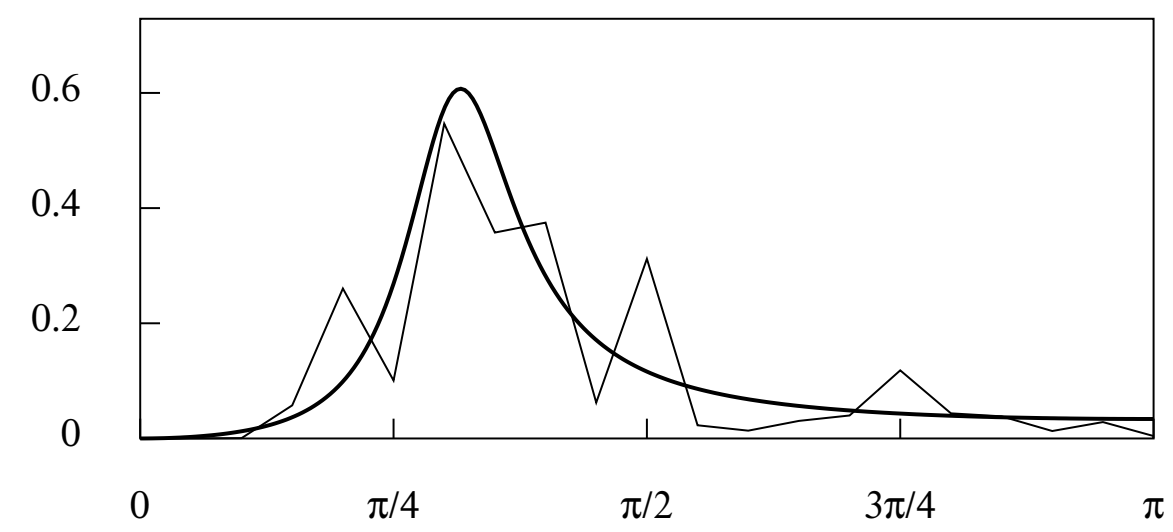

Figure 4. The periodogram of the de-noised data that have been filtered and subsampled at the rate of 1 observation in 4 , overlaid by the parametric spectrum of an estimated $\operatorname{ARMA}(2,1)$ model.

The spectral support of an ARMA process is the full Nyquist frequency interval $[0, \pi]$. Therefore, it is appropriate to dilate the spectral signature of the business cycle so that it fills the entire interval. This entails associating to each of the periodogram ordinates a higher frequency value. The frequencies are measured relative to the sampling interval. Therefore, they can be increased by increasing the length of the sampling interval.

In order to resample the data, it is usually necessary to reconstitute the underlying continuous trajectory. This can be achieved by a method of Fourier synthesis based on a version of equation (1) in which the coefficients associated with noisy elements, with frequencies in excess of the upper limit of the business cycle, have been set to zero.

The discrete temporal index, which is $t=0,1, \ldots, T-1$, can be replaced within equation (1) by a continuous variable $t \in[0, T)$ to create the continuous trajectory. This can be resampled at intervals of $\pi / \omega_{c}$ units of time. In the present example, wherein $\omega_{c}=\pi / 4$, the appropriate sampling interval is 4 units, which implies that only one in 4 of the points from the de-noised data is required; and there is no need to reconstitute the continuous trajectory in order to resample it. The effect of estimating an ARMA model with the de-noised and resampled data is shown in Figure 4.

\section{Sinc Function Interpolation and Fourier Interpolation}

The procedure for resampling the data has implicitly defined a continuous ARMA process powered by a continuous frequency-limited white-noise process. The stochastic differential equations that are commonly supposed to be the continuoustime analogues of the ARMA models are driven by the increments of a Wiener 
process. The latter is an accumulation of a continuous steam of infinitesimal impulses. Such impulses are unbounded in frequency. The Wiener process has the characteristic that, whatever the rate of sampling, the accumulations that occur within the sampling intervals will constitute a discrete-time white-noise process.

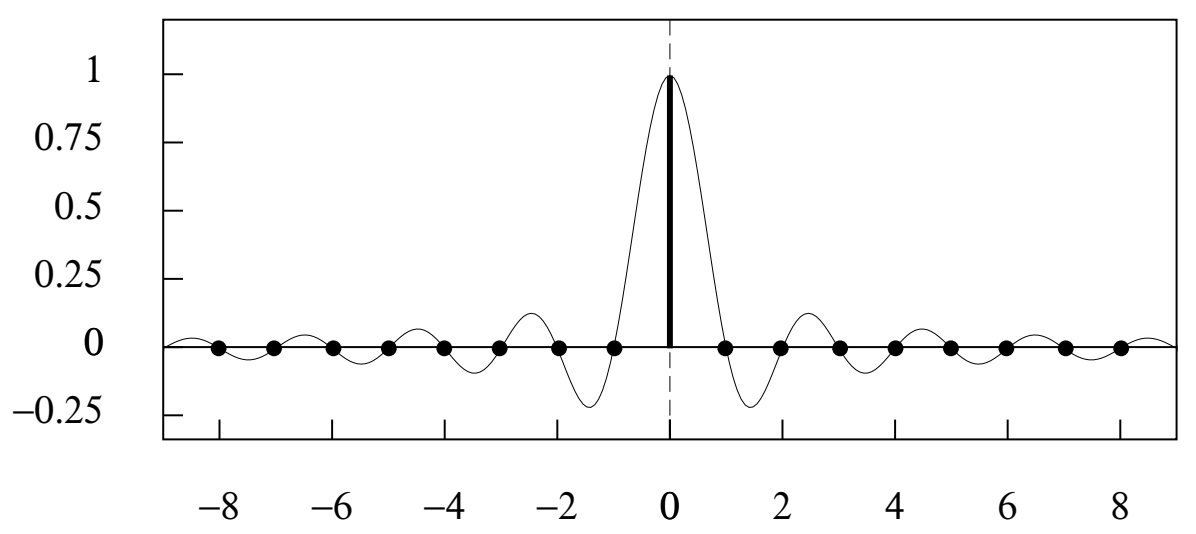

Figure 5. The sinc function wave-packet $\varphi(t)=\sin (\pi t) / \pi t$ comprising frequencies in the interval $[0, \pi]$.

In proposing a frequency-limited white noise, we resort to the sampling theorem. The theorem is commonly defined for square-integrable functions of time, defined of the the real line $\mathcal{R}=(-\infty, \infty)$, and limited in frequency to the interval $[-\pi, \pi]$.

The Fourier integral transform has the following expression in the time domain and the frequency domain:

$$
x(t)=\frac{1}{2 \pi} \int_{-\infty}^{\infty} \xi(\omega) e^{\mathrm{i} \omega t} d \omega \longleftrightarrow \xi(\omega)=\int_{-\infty}^{\infty} x(t) e^{-\mathrm{i} \omega t} d t .
$$

However, with the frequency limitation, this becomes

$$
x(t)=\frac{1}{2 \pi} \int_{-\pi}^{\pi} \xi_{S}(\omega) e^{\mathrm{i} \omega t} d \omega \longleftrightarrow \xi_{S}(\omega)=\sum_{k=-\infty}^{\infty} x_{k} e^{-\mathrm{i} k \omega},
$$

where $\left\{x_{k} ; k=0, \pm 1, \pm 2, \ldots\right\}$ is sampled at unit intervals from $x(t)$. Putting the RHS of (6) into the LHS and interchanging the order of integration and summation gives

$$
x(t)=\frac{1}{2 \pi} \sum_{k=-\infty}^{\infty} x_{k}\left\{\int_{-\pi}^{\pi} e^{\mathrm{i} \omega(t-k)}\right\} d \omega=\sum_{k=-\infty}^{\infty} x_{k} \varphi(t-k),
$$

where

$$
\varphi(t-k)=\frac{\sin \{\pi(t-k)\}}{\pi(t-k)}
$$

is the so-call sinc function. The RHS of equation (7) defines a sinc function interpolation.

The sinc function centred on $k=0$, which is illustrated in Figure 5, is formed by applying a bi-directional hyperbolic taper to an ordinary sine function. The 
succession of displaced sinc functions provides an orthonormal basis for the set of continuous functions that are limited in frequency to the Nyquist interval $[-\pi, \pi]$.

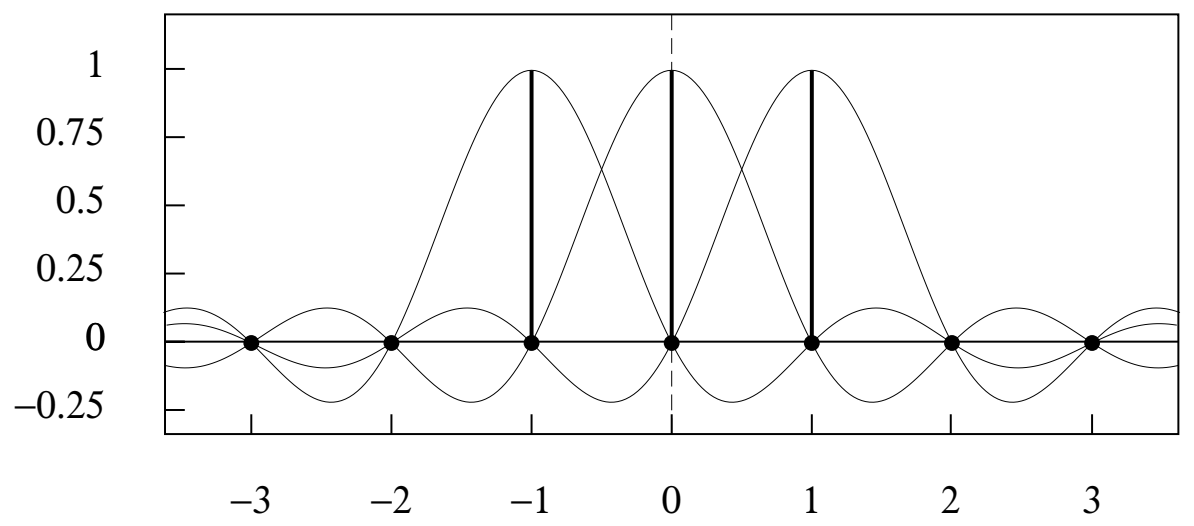

Figure 6. The wave packets $\varphi(t-k)$, which are bounded in frequency by $\pi$, suffer no mutual interference when $k \in\{0, \pm 1, \pm 2, \pm 3, \ldots\}$.

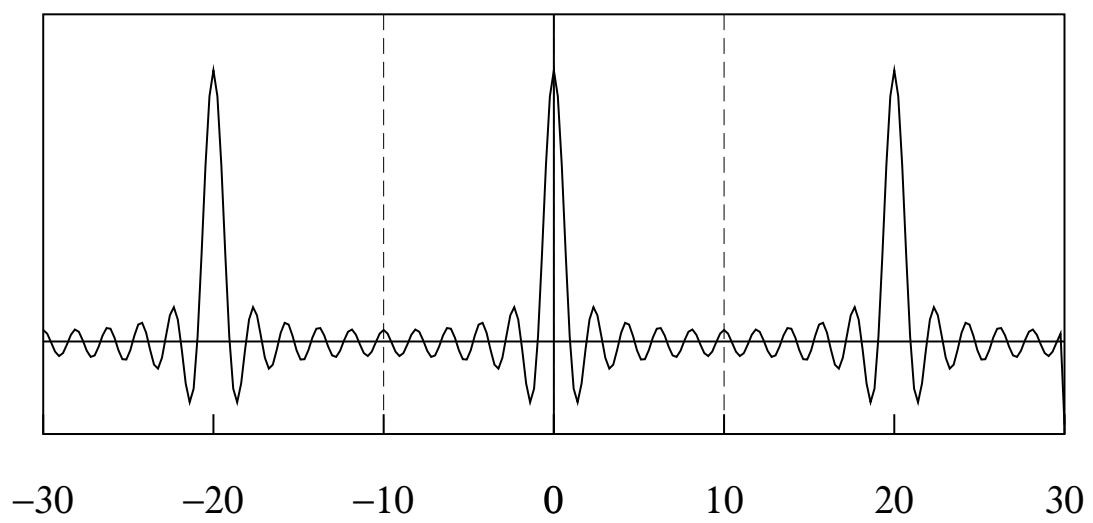

Figure 7. The Dirichlet function $\sin (\pi t) / \sin (2 \pi t / M)$ obtained from the inverse Fourier transform of a frequency-domain rectangle sampled at $M=21$ points.

Equation (7) implies a simple prescription for converting a data sequence into a continuous function that is limited in frequency to the Nyquist interval. Sinc function kernels are attached to each of the discrete-time ordinates, and the sum is taken of the scaled kernels. The values at the integer points are those of their associated kernels; and these values are not affected by the kernels at the other integer points. This feature is illustrated by Figure 6 .

A continuous-time white-noise forcing function can be formed by replacing the impulses of a discrete-time white-noise process by sinc functions scaled by the values of those impulses. If $\varepsilon_{t}=\varepsilon(t)$ and $\varepsilon_{s}=\varepsilon(s)$ are elements sampled at arbitrary points from the continuous frequency-limited white-noise forcing function, then their covariance is the sinc function

$$
C\left(\varepsilon_{t}, \varepsilon_{s}\right)=\sigma_{\varepsilon}^{2} \varphi(t-s)=\sigma_{\varepsilon}^{2} \varphi(\tau), \quad \tau=t-s,
$$

where $\sigma_{\varepsilon}^{2}$ is the variance parmeter. This result follows from recognising that $\varepsilon_{s}=$ $\varepsilon(\tau) \varepsilon_{t}+\eta$, where $\eta$ is uncorrelated with $\varepsilon_{t}$, and from the fact that $\varphi^{2}(\tau)=\varphi(\tau)$. 
The practicality of a sinc function synthesis is prejudiced by the fact that the support of the kernel functions is the entire real line $\mathcal{R}=(-\infty, \infty)$. A practical synthesis replaces the sinc function by the so-called Dirichlet kernel, which is a periodic or circular function formed by wrapping the sinc function around a circle of a circumference $T$, equal to the number of data points, and by adding the overlying ordinates. In this context, the data points to which the kernels are to be fixed are also to be regarded as a periodic or circular sequence.

Consider the discrete Fourier transform expressed as follows:

$$
x_{t}=\sum_{j=0}^{T-1} \xi_{j} e^{\mathrm{i} \omega_{j} t} \longleftrightarrow \xi_{j}=\frac{1}{T} \sum_{t=0}^{T-1} x_{t} e^{-\mathrm{i} \omega_{j} t} \quad \text { with } \quad \omega_{j}=\frac{2 \pi j}{T} .
$$

By putting the RHS of the latter into the LHS and commuting the two summations and allowing $t \in[0, T)$ to vary continuously, we get

$$
x(t)=\frac{1}{T} \sum_{k=0}^{T-1} x_{k}\left\{\sum_{j=0}^{T-1} e^{\mathrm{i} \omega_{j}(t-k)}\right\}=\sum_{k=0}^{T-1} x_{k} \varphi^{\circ}(t-k) .
$$

where

$$
\varphi^{\circ}(t)=\frac{1}{T} \sum_{j=-n}^{n} e^{\mathrm{i} \omega_{j} t}=\frac{\sin \left(\{T / 2\} \omega_{1} t\right)}{T \sin \left(\omega_{1} t / 2\right)} \quad \text { with } \quad n=\frac{T-1}{2} .
$$

is the periodic Dirichlet Kernel. An example is provided by Figure 7 .

Equation (11) implies that a sinc function interpolation of a finite data sequence that employs a sequence of Dirichelet kernels is equivalent to an interpolation based on a Fourier synthesis.

\section{Discrete-time and Continuous-time Models}

Whereas it is straightforward to derive a continuous version of an ARMA process (i.e. a CARMA process) by sinc function interpolation, we also require to represent it via a linear stochastic differential equation (an LSDE). The correspondence between difference equations and differential equations can be established by focusing, initially, on the first-order equations.

(In this paper, the acronym CARMA is reserved for the continuous-time linear stochastic differential equations that have the same frequency limitation as the corresponding discrete-time ARMA models. This is in spite of the common use of the acronym to denote continuous processes of unlimited frequency that are derived from ARMA models.)

The first-order autoregressive difference equation takes the form of

$$
y(t)=\mu y(t-1)+\varepsilon(t) \quad \text { or } \quad y(t)=\frac{\varepsilon(t)}{1-\mu L}=\sum_{\tau=0}^{\infty} \mu^{\tau} \varepsilon(t-\tau) .
$$

Here, $y(t)=\left\{y_{t} ; t=0 \pm 1, \pm 2 \ldots\right\}$ denotes a sequence, and $L$ is the lag operator such that $L y(t)=y(t-1)$. (However, $y(t)$ will be used, equally, to denote a 
function of a continuous-time index.) Also, the forcing function $\varepsilon(t)$ is a whitenoise sequence of independent and identically distributed random elements.

The first-order stochastic differential equation is denoted by

$$
\begin{gathered}
\frac{d y}{d t}=\kappa y(t)+\zeta(t) \quad \text { or } \\
y(t)=\frac{\zeta(t)}{D-\kappa}=\int_{0}^{\infty} e^{\kappa \tau} \zeta(t-\tau) d \tau=\int_{-\infty}^{t} e^{\kappa(t-\tau)} \zeta(\tau) d \tau
\end{gathered}
$$

where $D$ is the derivative operator such that $D x(t)=d x / d t$. Here, the forcing function $\zeta(t)$ is a continuous white-noise process. It is either the derivative of a Wiener process of else it is a frequency-limited process formed by associating sinc functions to the elements of a discrete white-noise sequence. It can be seen that $\mu^{\tau}$ and $e^{\kappa \tau}$ play the same role in the two equations, which is to diminish or to 'dampen' the effect of the impulses of the forcing functions as time elapses.

To convert the differential equation of (14) to the difference equation of (13), the integral on the interval $(-\infty, t]$ may be separated into two parts, which are the integrals over $(-\infty, t-1]$ and $(t-1, t]$ :

$$
\begin{aligned}
y(t) & =e^{\kappa} \int_{-\infty}^{t-1} e^{\kappa(t-1-\tau)} \zeta(\tau) d \tau+\int_{t-1}^{t} e^{\kappa(t-\tau)} \zeta(\tau) d \tau \\
& =\mu y(t-1)+\varepsilon(t) .
\end{aligned}
$$

We are interested, of course, in equations of higher orders. The $\operatorname{ARMA}(p, q)$ equation is denoted by

$$
\begin{gathered}
\left(1+\alpha_{1} L+\cdots+\alpha_{p} L^{p}\right) y(t)=\left(\beta_{0}+\beta_{1} L+\cdots+\beta_{p} L^{q}\right) \varepsilon(t) \\
\text { or } \quad \alpha(L) y(t)=\beta(L) \varepsilon(t) .
\end{gathered}
$$

Given that $p>q$ and that there are no repeated roots of $\alpha(z)=0$, the rational function $\beta(z) / \alpha(z)$ is amenable to a partial-fraction decomposition, which gives rise to the equation

$$
\begin{aligned}
y(t)=\frac{\beta(L)}{\alpha(L)} \varepsilon(t) & =\left\{\frac{d_{1}}{1-\mu_{1} L}+\frac{d_{2}}{1-\mu_{2} L}+\cdots+\frac{d_{p}}{1-\mu_{p} L}\right\} \varepsilon(t) \\
& =\sum_{\tau=0}^{\infty}\left\{d_{1} \mu_{1}^{\tau}+d_{2} \mu_{2}^{\tau}+\cdots+d_{p} \mu_{p}^{\tau}\right\} \varepsilon(t-\tau) .
\end{aligned}
$$

The linear stochastic differential equation of orders $p$ and $q<p$, denoted by $\operatorname{LSDE}(p, q)$, is specified by the equation

$$
\begin{aligned}
\left(\phi_{0} D^{p}+\phi_{1} D^{p-1}+\cdots+\phi_{p}\right) y(t) & =\left(\theta_{0} D^{q}+\theta_{1} D^{q-1}+\cdots+\theta_{q}\right) \zeta(t) \\
\text { or } \quad \phi(D) y(t) & =\theta(D) \zeta(t) .
\end{aligned}
$$

On the assumption that there are no repeated roots, it has the following partialfraction decomposition:

$$
\begin{aligned}
y(t)=\frac{\theta(D)}{\phi(D)} \zeta(t) & =\left\{\frac{c_{1}}{D-\kappa_{1}}+\frac{c_{2}}{D-\kappa_{2}}+\cdots+\frac{c_{p}}{D-\kappa_{p}}\right\} \zeta(t) \\
& =\int_{0}^{\infty}\left\{c_{1} e^{\kappa_{1} \tau}+c_{2} e^{\kappa_{2} \tau}+\cdots+c_{p} e^{\kappa_{p} \tau}\right\} \zeta(t-\tau) d \tau
\end{aligned}
$$




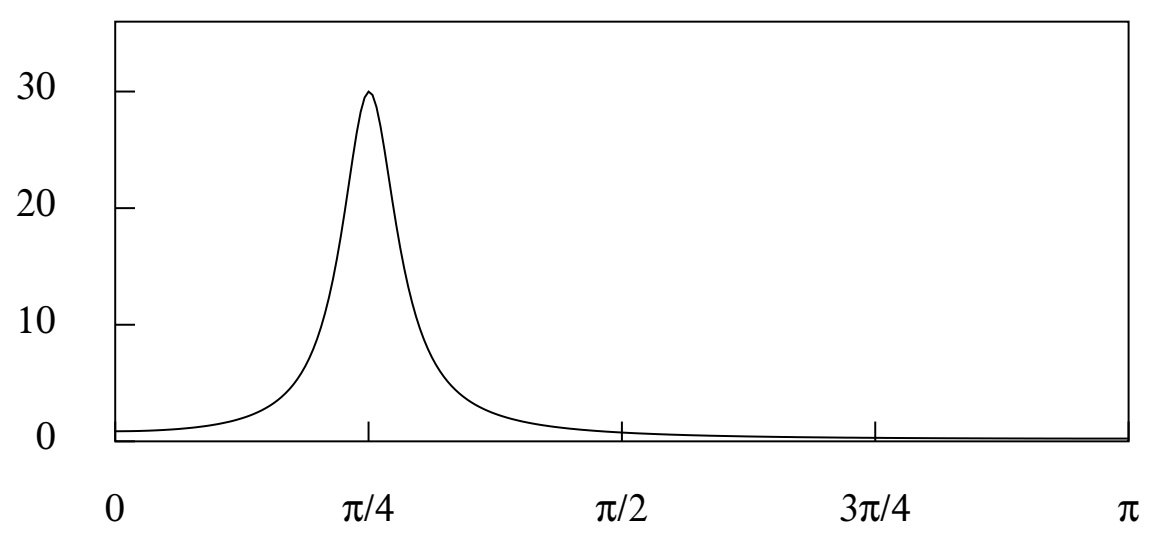

Figure 8. The spectrum of the $\operatorname{ARMA}(2,1)$ process $\left(1.0-1.273 L+0.81 L^{2}\right) y(t)=$ $(1-0.5 L) e(t)$.

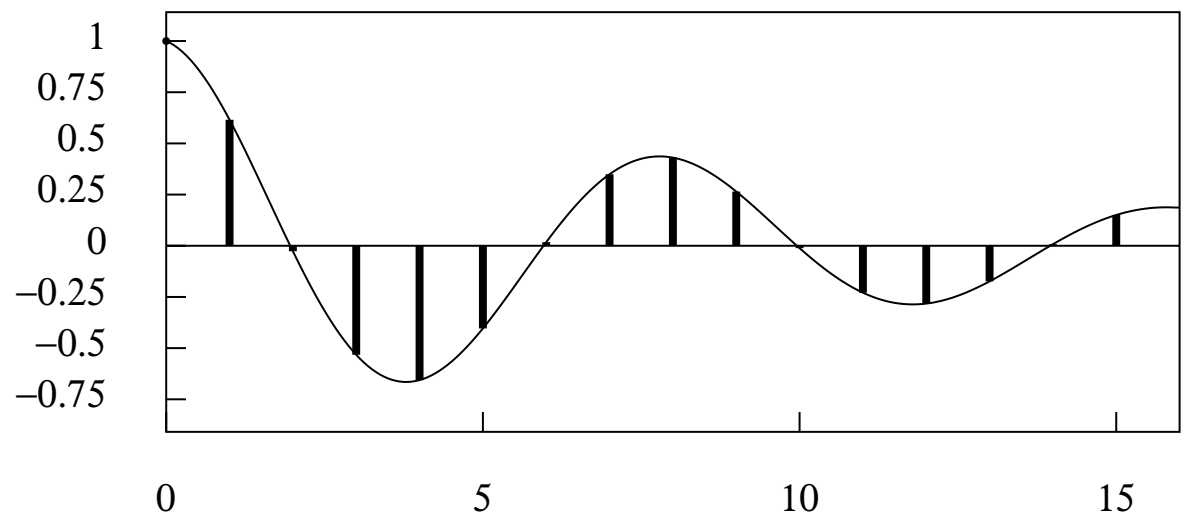

Figure 9. The discrete autocovariance sequence of the $\operatorname{ARMA}(2,1)$ process and the continuous autocovariance function of the corresponding $\operatorname{CARMA}(2,1)$ process.

\section{The ARMA Model and its Continuous-time CARMA Counterpart}

A correspondence can be established between the discrete-time ARMA model its continuous-time frequency-limited counterpart by invoking the principle of impulse invariance. This indicates that a sequence sampled at unit intervals from the impulse response function of the continuous system should be equal to the impulse response of the discrete-time system. This is only possible if the continuous system has the same frequency limitation as the discrete system, which is the present assumption.

Thus, at the integer values of $\tau$, the functions

$$
\psi(t)=c_{1} e^{\kappa_{1} \tau}+c_{2} e^{\kappa_{2} \tau}+\cdots+c_{p} e^{\kappa_{p} \tau}
$$

and

$$
\phi(\tau)=d_{1} \mu_{1}^{\tau}+d_{2} \mu_{2}^{\tau}+\cdots+d_{p} \mu_{2}^{\tau}
$$

should be equal. The equality can be achieved by setting $e^{\kappa_{j}}=\mu_{j}$ and $c_{j}=d_{j}$, for all $j$. The discrete-time ARMA model is driven by a white-noise process that is limited in frequency by the Nyquist value of $\pi$ radians per sample interval. Its 
direct continuous-time counterpart is a CARMA model, driven by a continuous frequency-limited white-noise process.

It is appropriate to adopt a CARMA model when there is clear evidence that the spectral density of the process is limited in frequency by the Nyquist value of $\pi$ radians per sample interval, at which point the function should be zero-valued. The evidence will be provided by the periodogram of the data. In cases where the limiting frequency of the process is less the $\pi$, the resampling procedures outlined in section 2 should be pursued before estimating the ARMA model.

Example 1. To illustrate the mapping from the discrete-time ARMA model to a continuous frequency-limited CARMA model, an $\operatorname{ARMA}(2,1)$ model is chosen with conjugate complex poles $\alpha \pm \mathrm{i} \beta=\rho \exp \{ \pm \mathrm{i} \theta\}$, where $\rho=\sqrt{\alpha^{2}+\beta^{2}}=0.9$ and $\theta=\tan ^{-1}(\beta / \alpha)=\pi / 4=45^{\circ}$. The moving-average component has a zero of 0.5 . The ARMA process generates prominent cycles of an average duration of roughly 8 periods.

The parameters of the resulting continuous-time CARMA model are displayed below, beside those of the ARMA model:

\begin{tabular}{ll}
\multicolumn{1}{c}{ ARMA } & CARMA \\
$\alpha_{0}=1.0$ & $\phi_{0}=1.0$ \\
$\alpha_{1}=-1.2728$ & $\phi_{1}=0.2107$ \\
$\alpha_{2}=0.8100$ & $\phi_{2}=0.6280$ \\
$\beta_{0}=1.0$ & $\theta_{0}=1.0$ \\
$\beta_{1}=-0.5$ & $\theta_{1}=0.2737$
\end{tabular}

The spectral density function of the ARMA process is illustrated in Figure 8. Here, it will be observed that the function is virtually zero at the limiting Nyquist frequency of $\pi$. Therefore, it is reasonable to propose that the corresponding continuous-time model should be driven by a white-noise forcing function that is bounded by the Nyquist frequency.

The spectral density function of the CARMA process is the integral Fourier transform of the continuous autocovariance function, whereas the spectral density function of the ARMA process is the discrete Fourier transform of the autocovariance sequence. The frequency limitation of the CARMA process means that there is no aliasing in the sampling process. Therefore, the two spectra are identical.

In Figure 9, the discrete autocovariance function of the ARMA process is superimposed on the continuous autocovariance function of the CARMA process. The former has been generated by a recursive procedure. The latter has been generated by an analytic equation, to be presented below as equation (22), wherein the index $\tau$ of the lags varies continuously.

A principle of autocovariance equivalence is satisfied, whereby the values sampled at the integer points from the continuous-time autocovariance function are equal to those of the discrete-time function. 


\section{Stochastic Differential Equations Driven by Wiener Processes}

The white-noise forcing function of a conventional linear stochastic differential equation (LSDE) is the derivative of a Wiener process. The latter process consists of a continuous steam of infinitesimal impulses. Since a pure impulse is unbounded in frequency, so too is the forcing function.

The concept of a pure impulse is problematic from a physical point of view, since it implies a discrete and instantaneous change in momentum. The problem of unbounded frequencies can be mitigated, if not completely overcome, in the context of an LSDE, since its transfer function may impose a sufficient attenuation on the higher frequencies for the effect to be a virtual frequency limitation.

Whenever the spectral density function of an ARMA model has a significant value at the Nyquist frequency of $\pi$, there can be a reasonable supposition that the underlying continuous process has a frequency range that extends beyond the Nyquist limit. Therefore, it may be appropriate to adopt an LSDE with an unbounded forcing function as the continuous-time counterpart of the ARMA model.

In translating from an ARMA model to such an LSDE, it is no longer appropriate to invoke the principle of impulse invariance. Instead, the principle of autocovariance equivalence that was enunciated by Bartlett (1946) must be adopted. The principle asserts that the parameters of the LSDE should be chosen so that its autocovariance function matches the autocovariance function of the ARMA model at the integer lags.

The autocovariance function of an ARMA model can be derived from its impulse response function, represented by equation (21). It takes the form of

$$
\begin{aligned}
\gamma^{d}(\tau) & =\sigma_{\varepsilon}^{2} \sum_{j=0}^{\infty}\left(\sum_{k=1}^{p} d_{k} \mu_{k}^{j}\right)\left(\sum_{k=1}^{p} d_{k} \mu_{k}^{j+\tau}\right) \\
& =\sigma_{\varepsilon}^{2} \sum_{i=1}^{p}\left\{\sum_{j=1}^{p} \frac{d_{i} d_{j}}{1-\mu_{i} \mu_{j}}\right\} \mu_{i}^{\tau} .
\end{aligned}
$$

The autocovariance function $\gamma^{c}(\tau)$ of the continuous-time LSDE process is also found via its impulse response function. It is assumed that the autocovariance of the white-noise forcing function at lag $\tau$ is

$$
E\{\zeta(t) \zeta(t-\tau)\}=\delta(\tau) \sigma_{\zeta}^{2}
$$

where $\delta(\tau)$ is Dirac's delta function. Then,

$$
\begin{aligned}
\gamma^{c}(\tau) & =E\{y(t) y(t-\tau)\} \\
& =E\left\{\int_{0}^{\infty} \psi(u) \zeta(t-u) d u \int_{0}^{\infty} \psi(v) \zeta(t-\tau-v) d v\right\} \\
& =\sigma_{\zeta}^{2} \int_{0}^{\infty} \psi(v) \psi(v+\tau) d v
\end{aligned}
$$


Substituting the expression of (20) for the continuous-time impulse response function $\psi(t)$ into equation $(24)$ gives

$$
\begin{aligned}
\gamma^{c}(\tau)=\sigma_{\zeta}^{2} \int_{0}^{\infty} \psi(t) \psi(t+\tau) d t & =\sigma_{\zeta}^{2} \sum_{i} \sum_{j}\left\{c_{i} c_{j} \int_{0}^{\infty} e^{\left(\kappa_{i}+\kappa_{j}\right) t+\kappa_{i} \tau} d t\right\} \\
& =\sigma_{\zeta}^{2} \sum_{i}\left\{\sum_{j} c_{i} c_{j} \frac{-e^{\kappa_{i} \tau}}{\kappa_{i}+\kappa_{j}}\right\} .
\end{aligned}
$$

This expression, which is liable to contain complex-valued terms, may be rendered in real terms by coupling the various conjugate complex terms.

In translating from the ARMA model to the LSDE, there continues to be a one-to-one correspondence between the poles of the two systems. If a complex pole of the ARMA model takes the form of

$$
\mu=\alpha+\mathrm{i} \beta=\rho\{\cos (\omega)+\mathrm{i} \sin (\omega)\}=\rho e^{\mathrm{i} \omega},
$$

with

$$
\rho=\sqrt{\alpha^{2}+\beta^{2}} \quad \text { and } \quad \omega=\tan ^{-1}\left(\frac{\beta}{\alpha}\right),
$$

then the corresponding pole of the LSDE and of the CARMA differential equation is

$$
\kappa=\ln (\mu)=\ln (\rho)+\mathrm{i} \omega=\delta+\mathrm{i} \omega,
$$

with $\delta \in(-\infty, 0)$, which puts it in the left half of the $s$-plane, as is necessary for the stability of the system.

The principle of autocovariance equivalence can be expressed via the equation

$$
\gamma_{\tau}^{c}\{\kappa(\mu), c\}=\gamma_{\tau}^{d}(\mu, d) \quad \text { for } \quad \tau \in\{0, \pm 1, \pm 2, \ldots\}
$$

Then, the parameters of the LSDE can be derived once a value of $c=\left[c_{1}, c_{2}, \ldots, c_{p}\right]$ of the vector of the numerator parameters of (19) has been found that satisfies this equation. The value of $c$ can be found by using an optimisation procedure to find the zeros of the function

$$
z(c)=\sum_{\tau=0}^{p}\left\{\gamma_{\tau}^{c}(c)-\gamma_{\tau}^{d}\right\}^{2} .
$$

As Söderström $(1990,1991)$, and others have noted, there are ARMA models for which there are no corresponding LSDE's. The present procedure for translating from an ARMA model to an LSDE reveals such cases by its failure to find a zerovalued minimum of the criterion function. However, it can be relied upon to find the LSDE most closely related to the ARMA model.

The principle of autocovariance equivalence also indicates a way in which an ARMA model can be found to correspond to a given LSDE. The ARMA model is commonly described as the exact or equivalent discrete linear model (EDLM). 
The autocovariance generating function of an ARMA model is

$$
\gamma^{d}(z)=\sigma_{\varepsilon}^{2} \frac{\beta(z) \beta\left(z^{-1}\right)}{\alpha(z) \alpha\left(z^{-1}\right)}
$$

whereas the $z$-transform of the elements $\gamma_{\tau}^{c} ; \tau \in\{0, \pm 1, \pm 2, \ldots\}$ sampled from the autocovariance function of the LSDE may be denoted by $\gamma^{c}(z)$. Putting the latter in place of $\gamma^{d}(z)$ and rearranged the equation gives

$$
\sigma_{\varepsilon}^{2} \beta(z) \beta\left(z^{-1}\right)=\alpha(z) \gamma^{c}(z) \alpha\left(z^{-1}\right)
$$

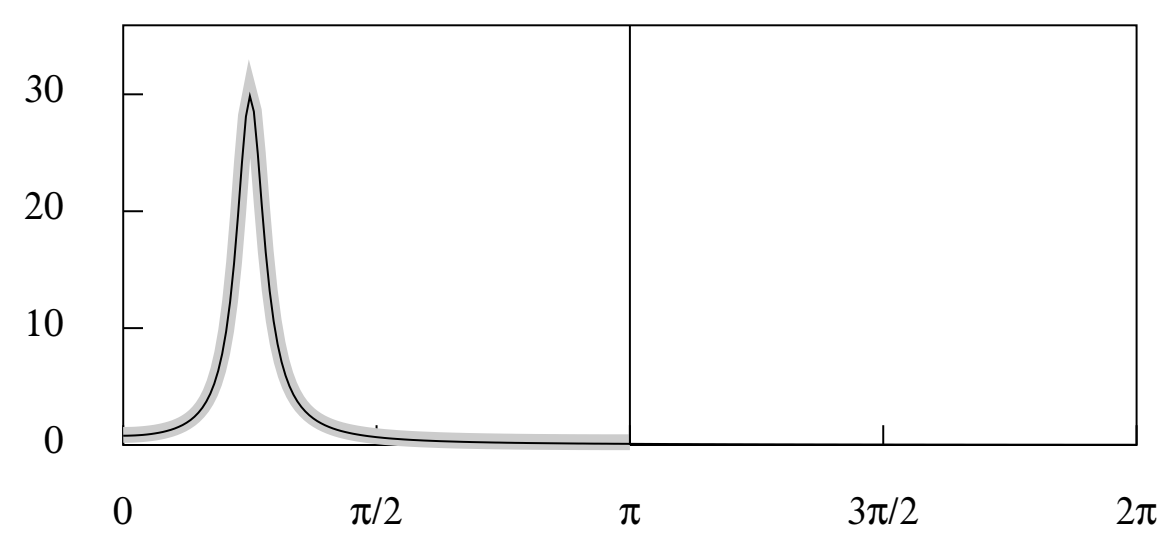

Figure 10. The spectrum of the $\operatorname{LSDE}(2,1)$ corresponding to the $\operatorname{ARMA}(2,1)$ model of Example 1 plotted on top of the spectrum of that model, represented by the thick grey line. The two spectra virtually coincide over the interval $[0, \pi]$.

Since the discrete-time autoregressive parameters within $\alpha(z)$ can be inferred from those of the LSDE, only the moving-average parameters within $\beta(z)$ and the variance $\sigma_{\varepsilon}^{2}$ need to be derived from equation (32). They can be obtained via a Cramér-Wold factorisation of the LHS.

Example 2. The mapping from the discrete-time ARMA model to a continuoustime LSDE model can be illustrated, in the first instance, with the $\operatorname{ARMA}(2,1)$ model of Example 1.

The parameters of the corresponding $\operatorname{LSDE}(2,1)$ model are obtained by using the procedure of Nelder and Mead (1965) to find the minimum of the criterion function of (30), where it is assumed that the variance of the forcing function is $\sigma_{\zeta}^{2}=1$. The minimands $a, b$ of the criterion function are from the numerator coefficients $c, c^{*}=a \pm \mathrm{i} b$ of the partial-fraction decomposition of the $\operatorname{LSDE}(2,1)$ transfer function.

There are four points that correspond to zero-valued minima, where the ordinates of the discrete and continuous autocovariance functions coincide at the integer lags. These points, together with the corresponding moving-average parameters, 
are as follows:

\begin{tabular}{ccccc}
\hline & $a$ & $b$ & $\theta_{0}$ & $\theta_{1}$ \\
\hline$(1)$ & -0.4544 & 0.2956 & -0.9088 & 0.5601 \\
$(2)$ & 0.4544 & 0.4175 & 0.9088 & 0.5601 \\
$(3)$ & -0.4544 & -0.4174 & -0.9088 & -0.5601 \\
$(4)$ & 0.4544 & -0.2956 & 0.9088 & -0.5601
\end{tabular}

Here, the parameter values of (1) and (4) are equivalent, as are those of (2) and (3). Their difference is a change of sign, which can be eliminated by normalising $\theta_{0}$ at unity and by adjusting variance of the forcing function accordingly.

The miniphase condition, which corresponds to the invertibility condition of a discrete-time model, requires the zeros to be in the left half of the $s$-plane. Therefore, (2) and (3) on the $N E-S W$ axis are the chosen pair.

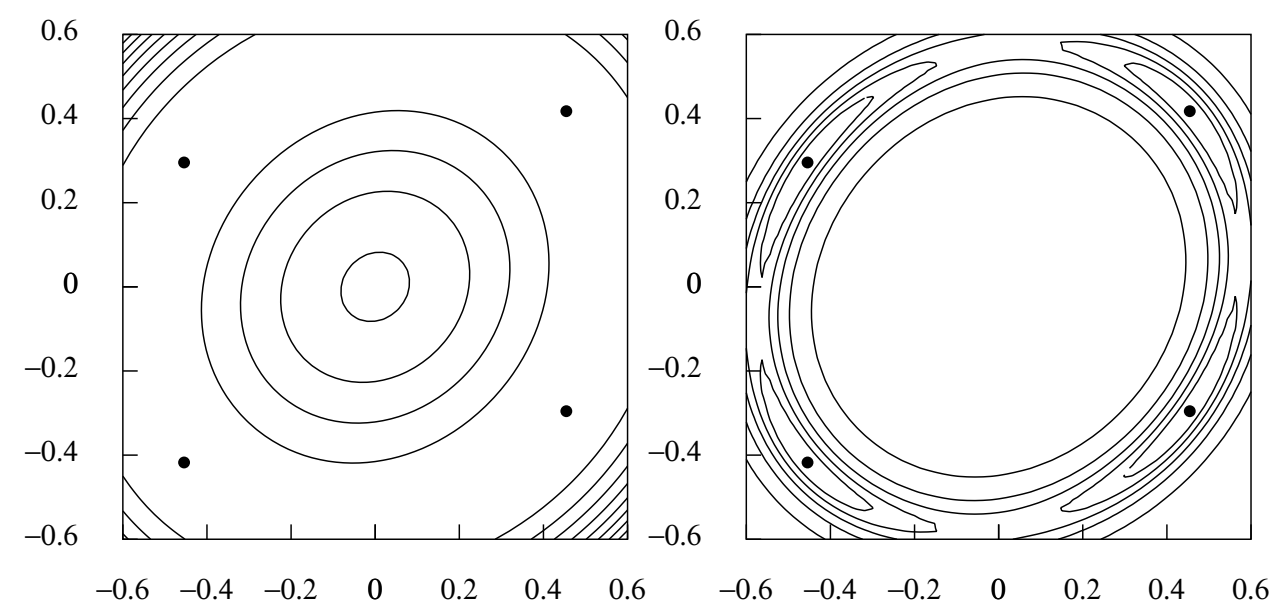

Figure 11. Left The contours of the criterion function $z=z(a, b)$ together with the minimising values, marked by black dots. Right The contours of the function $q=1 /(z+d)$.

These estimates of the $\operatorname{LSDE}(2,1)$ are juxtaposed below with those of the CARMA $(2,1)$ model derived from the same ARMA model:

\begin{tabular}{ll}
\multicolumn{1}{c}{ CARMA } & LSDE \\
$\phi_{0}=1.0$ & $\phi_{0}=1.0$ \\
$\phi_{1}=0.2107$ & $\phi_{1}=0.2107$ \\
$\phi_{2}=0.6280$ & $\phi_{2}=0.6280$ \\
$\theta_{0}=1.0$ & $\theta_{0}=0.9088$ \\
$\theta_{1}=0.2737$ & $\theta_{1}=0.5601$
\end{tabular}

The autoregressive parameters of the CARMA model and of the LSDE model are, of course, identical. However, there is a surprising disparity between the two 
sets of moving-average parameters. Nevertheless, when they are superimposed on the same diagram - which is Figure 10 - the spectra of the two models are seen virtually to coincide. Moreover, the parameters of the ARMA model can be recovered exactly from those of the LSDE by an inverse transformation.

The explanation for this outcome is to be found in the remarkable flatness of the criterion function in the vicinity of the minimising points, which are marked on both sides of Figure 11 by black dots. The flatness implies that a wide spectrum of the parameter values of the LSDE will give rise to almost identical autocovariance functions and spectra.

The left side of Figure 11 shows some equally-spaced contours of the $z$-surface of the criterion function, which are rising from an annulus that contains the minima. The minima resemble small indentations in the broad brim of a hat.

The right side of Figure 11, which is intended to provide more evidence of the nature of the criterion function in the vicinity of the minima, shows the contours of the function $q=1 /(z+d)$, where $d$ is a small positive number that prevents a division by zero. We set $d=(X-R M) /(R-1)$, where $M=\min (z), X=$ $\max (z)$ and where $R=\max (q) / \min (q)=60$. The extended lenticular contours surrounding the minimising points of the criterion function, which have become maxima in this diagram, are a testimony to the virtual equivalence of a wide spectrum of parameter values.

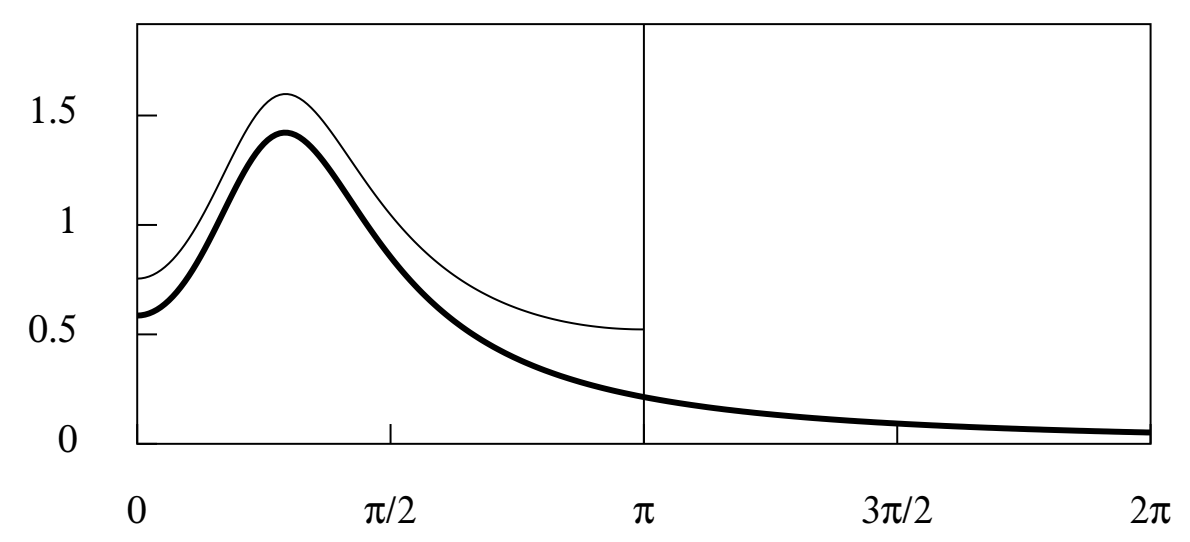

Figure 12. The spectrum of the revised ARMA model superimposed on the spectrum of the derived LSDE, described by the heavier line.

Example 3. A variant to the $\operatorname{ARMA}(2,1)$ model is one that has a pair of complex conjugate poles $\rho \exp \{ \pm \mathrm{i} \theta\}$ with the same argument as before, which is $\theta=\pi / 4=$ $45^{\circ}$, and with a modulus that has been reduced to $\rho=0.5$. The model retains the zero of 0.5. The ARMA parameters and those of the corresponding LSDE are as follows: 


$\begin{array}{ll}\text { ARMA } & \text { LSDE } \\ \alpha_{0}=1.0 & \phi_{0}=1.0 \\ \alpha_{1}=-0.7071 & \phi_{1}=1.3863 \\ \alpha_{2}=0.2500 & \phi_{2}=1.0973 \\ \beta_{0}=1.0 & \theta_{0}=1.5012 \\ \beta_{1}=-0.5 & \theta_{1}=0.8905\end{array}$

Figure 12 shows the spectral density functions of the LSDE and of the ARMA model superimposed on same diagram. The spectrum of the LSDE extends far beyond the Nyquist frequency of $\pi$, which is the limiting ARMA frequency.

The ARMA process, which is to be regarded as a sampled version of the LSDE, is seen to suffer from a high degree of aliasing, whereby the spectral power of the LSDE that lies beyond the Nyquist frequency is mapped into the Nyquist interval $[-\pi, \pi]$, with the effect that the profile of the ARMA spectrum is raised considerably. On this basis, it can be asserted that the ARMA model significantly misrepresents the underlying continuous-time process.

\section{Summary and Conclusions}

The intention of this paper has been to clarify the relationship between unconditional linear stochastic models in discrete and continuous time, and to provide secure means of computing the continuous models. The importance of an awareness of the frequency-domain characteristics of the forcing functions has been emphasised.

Example 1 has demonstrated a straightforward way of deriving a frequencylimited stochastic differential equation that correspond to a discrete-time ARMA model. This has been described as a continuous-time CARMA model.

This model is a valid representation of the underlying process only if the maximum frequency of that process corresponds to the limiting frequency of the ARMA model, which is $\pi$ radians per sampling interval. To ensure that this is the case, it may be necessary to reconstitute the continuous trajectory and to resample it at a reduced rate.

The forcing function of a conventional linear stochastic differential equation, or LSDE, which consists of the increments of a Wiener process, is unbounded in frequency. This seems to be inappropriate to a model of a frequency-limited process. Nevertheless, the transfer function of the LSDE may impose a radical attenuation on the higher frequencies that implies a virtual frequency limitation. Example 2 has illustrated such a case.

Example 3 has shown the aliasing effects that occur when the forcing function has no frequency limit and when the ARMA transfer function imposes only a weak attenuation on the high-frequency elements. This provides a ready justification for adopting an LSDE as the continuous-time counterpart of the ARMA model.

The spectral density function of the ARMA model will be formed by wrapping the spectrum of the LSDE around a circle of circumference $2 \pi$ and by adding the overlying ordinates. In this way, the spectral component of frequencies in excess of the Nyquist value are mapped into the interval $[-\pi, \pi]$ to produce a discrete-time 
spectrum that may depart significantly from the continuous-time parent spectrum, as represented by the derived LSDE. This is seen in Figure 12.

The methods for translating from an ARMA model to a continuous-time model, which may be a frequency-limited CARMA model or an LSDE model that is without an ostensible frequency restriction, have been realised in the computer program CONCRETE.PAS, which available at the the author's website, where both the compiled program and its code can be found.

An associated program CONTEXT.PAS, which plots the contour map of the surface of the criterion function that is employed in matching the autocovariance function of the $\operatorname{LSDE}(2,1)$ to that of the $\operatorname{ARMA}(2,1)$ model, is also available.

\section{References.}

Bartlett, M.S., (1946), On the Theoretical Specification and Sampling Properties of Autocorrelated Time Series, Supplement to the Journal of the Royal Statistical Society, 8, 27-41.

Hodrick, R.J., and E.C. Prescott, (1980), Postwar U.S. Business Cycles: An Empirical Investigation, Working Paper, Carnegie-Mellon University, Pittsburgh, Pennsylvania.

Hodrick R.J., and E.C. Prescott, (1997), Postwar U.S. Business Cycles: An Empirical Investigation, Journal of Money, Credit and Banking, 29, 1-16.

Leser, C.E.V., (1961), A Simple Method of Trend Construction, Journal of the Royal Statistical Society, Series B, 23, 91-107.

Nelder, J.A., and R. Mead, (1965), A Simplex Method for Function Minimization, Computer Journal, 7, 308-313.

Nyquist, H., (1924), Certain Factors Affecting Telegraph Speed, Bell System Technical Journal 3, 324-346.

Nyquist, H., (1928), Certain Topics in Telegraph Transmission Theory, Transactions of the AIEE, 47, 617-644. Reprinted in 2002, Proceedings of the IEEE, 90, 280-305.

Pollock D.S.G., (2017), Linear Stochastic Models in Discrete and Continuous Time, Unpublished paper.

Shannon, C.E., (1949), Communication in the Presence of Noise, Proceedings of the Institute of Radio Engineers, 37, 10-21. Reprinted in 1998, Proceedings of the IEEE, 86, 447-457.

Söderström, T., (1990), On Zero Locations for Sampled Stochastic Systems, IEEE Transactions on Automatic Control, 35, 1249-1253.

Söderström, T., (1991), Computing Stochastic Continuous-Time Models from ARMA Models, International Journal of Control, 53, 1311-1326. 